\title{
Quantum Fisher information for states in exponential form
}

\author{
Zhang Jiang ${ }^{1}$ \\ ${ }^{1}$ Center for Quantum Information and Control, University of New Mexico, \\ MSC07-4220, Albuquerque, New Mexico 87131-0001, USA
}

(Dated: April 1, 2014)

\begin{abstract}
We derive explicit expressions for the quantum Fisher information and the symmetric logarithmic derivative (SLD) of a quantum state in the exponential form $\rho=\exp (G)$; the SLD is expressed in terms of the generator $G$. Applications include quantum-metrology problems with Gaussian states and general thermal states. Specifically, we give the SLD for a Gaussian state in two forms, in terms of its generator and its moments; the Fisher information is also calculated for both forms. Special cases are discussed, including pure, degenerate, and very noisy Gaussian states.
\end{abstract}

PACS numbers: 03.65.Ta, 03.67.-a, 06.20.-f

\section{INTRODUCTION}

Quantum metrology studies the limit to the accuracy, set by quantum mechanics, with which physical quantities can be estimated by measurements. The basic idea is to determine an unknown parameter $\theta$ by probing a quantum state that depends on the parameter. Quantum metrology is important for various purposes, which include improving time and frequency standards [1, 2], detecting gravitational waves [3, 4], interferometry based on interacting systems [5, 6], and magnetometry $[7,8]$.

A standard scenario for quantum parameter estimation is to put a known initial state $\rho_{\text {in }}$ through a quantum channel $\mathcal{E}_{\theta}$ that impresses $\theta$ on the system; the output state $\rho(\theta)=\mathcal{E}_{\theta}\left(\rho_{\text {in }}\right)$ is then subjected to a measurement. The goal is to find the optimal measurement strategy so that as much information as possible about $\theta$ is acquired. Although it is hard to solve the most general problem exactly, bounds on how accurately one can estimate a parameter can be obtained [9-12].

In classical parameter estimation theory, the Cramér-Rao bound (CRB) expresses a lower bound on the variance of an unbiased estimator $\theta_{\text {est }}$,

$$
\operatorname{var}\left(\theta_{\text {est }}\right) \geq \frac{1}{\mathcal{I}_{c}(\theta)}
$$

where $\mathcal{I}_{c}(\theta)$ is the classical Fisher information [13]. Fisher's theory says that maximum likelihood estimation achieves the CRB asymptotically for large number of trials [14, 15]. For the quantum case, it was shown, in [16], that there exists an optimal quantum measurement whose classical Fisher information, obtained from the measurement outcomes, achieves the quantum Fisher information [16-19],

$$
\mathcal{I}(\theta)=\operatorname{tr}\left(\rho(\theta) L^{2}(\theta)\right) .
$$

Thus the inverse of the quantum Fisher information gives the quantum CRB on the variance of an estimator. The (Hermitian) operator $L(\theta)$, in Eq. (1.2), is the symmetric logarithmic derivative (SLD), defined implicitly by

$$
\frac{d \rho(\theta)}{d \theta}=\frac{1}{2}\{L(\theta), \rho(\theta)\},
$$

where the brackets denote the anticommutator. Knowing the SLD allows one to obtain not only the Fisher information but also the optimal measurement scheme.

Any full rank quantum state $\rho(\theta)$ can be written in exponential form,

$$
\rho(\theta)=e^{G(\theta)},
$$

with the normalization absorbed into $G(\theta)$. The case that $\rho(\theta)$ is not invertible can be handled as a limit in which some eigenvalues of $G(\theta)$ go to minus infinity. The form (1.4) is useful when $G(\theta)$ takes a simple form, examples being Gaussian states and general thermal states. Gaussian states are important because of their appealing properties for quantum-metrology tasks [20-22] and their accessibility both to experimentalists and theorists. Thermal states are also useful for quantum-metrology tasks for at least two reasons: (i) The initial state is often a thermal state $\rho_{\text {in }}=e^{-\beta H} / Z$, and the simple exponential form is preserved by a unitary channel $U_{\theta}$. (ii) We can infer the temperature 
and the chemical potential by measuring the state $\rho(\theta)=e^{-\beta(H-\mu N)} / Z$, after the system is brought to thermodynamic equilibrium with a reservoir [23].

In Sec. II, we consider the SLD for a quantum state in the exponential form (1.4). We show that the SLD can be expanded into a weighted sum of $d G / d \theta$ and its recursive, nested commutators with $G$. Simple expressions of the quantum Fisher information and the SLD are given in the basis where $G$ is diagonalized. In Sec. III, we apply the results of Sec. II to Gaussian states, and an explicit expression of the SLD in terms of the generator is derived. In Sec. IV, also for Gaussian states, the SLD and the quantum Fisher information are given in terms of the moments of position and momentum operators (or of creation and annihilation operators).

\section{QUANTUM FISHER INFORMATION FOR STATES IN EXPONENTIAL FORM}

A useful expression (see Eq. (2.1) of Ref. [24]) for density operators of the exponential form (1.4) is

$$
\dot{\rho}=\int_{0}^{1} e^{s G} \dot{G} e^{(1-s) G} d s,
$$

where an overdot denotes a derivative with respect to $\theta$. We now use the nested-commutator relation

$$
\begin{aligned}
e^{G} A e^{-G} & =A+[G, A]+\frac{1}{2 !}[G,[G, A]]+\cdots \\
& =\sum_{n=0}^{\infty} \frac{1}{n !} \mathcal{C}^{n}(A)=e^{\mathcal{C}}(A)
\end{aligned}
$$

where $\mathcal{C}^{n}(A)$, a linear operation on $A$, denotes the $n$ th-order nested commutator $[G, \ldots,[G, A]]$, with $\mathcal{C}^{0}(A)=A$. Applying this relation to the expression (2.1), we get

$$
\begin{aligned}
\dot{\rho} \rho^{-1} & =\dot{G}+\frac{1}{2 !}[G, \dot{G}]+\frac{1}{3 !}[G,[G, \dot{G}]]+\cdots \\
& =\sum_{n=0}^{\infty} \frac{1}{(n+1) !} \mathcal{C}^{n}(\dot{G})=h(\mathcal{C})(\dot{G})
\end{aligned}
$$

where $h$ is the generating function of the expansion coefficients in Eq. (2.3),

$$
h(t)=1+\frac{t}{2 !}+\frac{t^{2}}{3 !}+\cdots=\frac{e^{t}-1}{t} .
$$

Using the definitions (1.3) and (1.4), we also have

$$
\begin{aligned}
\dot{\rho} \rho^{-1} & =\frac{1}{2}\left(L+e^{G} L e^{-G}\right) \\
& =\frac{1}{2}\left(L+\sum_{n=0}^{\infty} \frac{1}{n !} \mathcal{C}^{n}(L)\right)=r(\mathcal{C})(L),
\end{aligned}
$$

where the generating function is $r(t)=\left(e^{t}+1\right) / 2$. Suppose that the SLD adopts the form,

$$
L=\sum_{n=0}^{\infty} f_{n} \mathcal{C}^{n}(\dot{G})=f(\mathcal{C})(\dot{G}),
$$

where the to be determined generating function $f$ is specified by

$$
f(t)=f_{0}+f_{1} t+f_{2} t^{2}+\cdots .
$$

By putting Eq. (2.6) into Eq. (2.5), we have

$$
\dot{\rho} \rho^{-1}=r(\mathcal{C})[f(\mathcal{C})(\dot{G})]=r \cdot f(\mathcal{C})(\dot{G}),
$$


where $r \cdot f$ is the product of the two functions, and we use the identity $\mathcal{C}^{n}\left(\mathcal{C}^{m}(A)\right)=\mathcal{C}^{n+m}(A)$. Comparing Eq. $(2.8)$ with Eq. (2.3), we have the relation among the generating functions,

$$
f(t)=\frac{h(t)}{r(t)}=\frac{\tanh (t / 2)}{t / 2}=\sum_{n=0}^{\infty} \frac{4\left(4^{n+1}-1\right) B_{2 n+2}}{(2 n+2) !} t^{2 n},
$$

where $B_{2 n+2}$ is the $(2 n+2)$ th Bernoulli number. Comparing Eqs. (2.7) with (2.9), we have

$$
f_{n}= \begin{cases}\frac{4\left(4^{n / 2+1}-1\right) B_{n+2}}{(n+2) !}, & \text { for even } n \\ 0, & \text { for odd } n\end{cases}
$$

The vanishing of the odd-order $f_{n} \mathrm{~s}$ is a consequence of the Hermiticity of $L$, which makes $f(t)$ an even function.

The first four nonzero coefficients $f_{n}$ are

$$
f_{0}=1, \quad f_{2}=-\frac{1}{12}, \quad f_{4}=\frac{1}{120}, \quad f_{6}=-\frac{34}{8 !} .
$$

Although it appears that the $f_{n}$ s become negligible very fast, they revive at larger $n$, and the radius of convergence of the power series (2.7) is $t<\pi$. This limits the usefulness of the expansion (2.6); it is divergent when the difference between any two eigenvalues of $G$ is greater than or equal to $\pi$. Fortunately, in many real problems, the recursive commutators in Eq. (2.6) either terminate or repeat, enabling us to find an exact solution. In the latter case, we can use analytic continuation to extend the result (2.6) beyond the domain of convergence.

Suppose that we work in the basis $\left|e_{j}\right\rangle$ where $G$ is diagonal, i.e., $G\left|e_{j}\right\rangle=g_{j}\left|e_{j}\right\rangle$. This basis generally changes with $\theta$, so we are considering here, as in the rest of this section, a particular value of $\theta$. In this basis, Eq. (2.6) is equivalent to

$$
L_{j k}=\left\langle e_{j}|L| e_{k}\right\rangle=f\left(g_{j}-g_{k}\right) \dot{G}_{j k}
$$

The domain of Eq. (2.12) is not restricted to the radius of convergence, $g_{j}-g_{k}<\pi$; it is well defined for any $G$, which is an example of analytic continuation. Using Eq. (2.3), we have

$$
\dot{\rho}_{j k}=\left\langle e_{j}|\dot{\rho}| e_{k}\right\rangle=e^{g_{k}} h\left(g_{j}-g_{k}\right) \dot{G}_{j k}
$$

and Eq. (2.12) can be converted to a formula familiar from Ref. [16],

$$
L_{j k}=\frac{\dot{\rho}_{j k}}{e^{g_{k}} r\left(g_{j}-g_{k}\right)}=\frac{2 \dot{\rho}_{j k}}{\rho_{j j}+\rho_{k k}},
$$

where $\rho_{j j}=e^{g_{j}}$. This formula follows directly from the definition (1.3) of the SLD.

The Fisher information can now be calculated directly in this same basis,

$$
\mathcal{I}=\sum_{j, k} e^{g_{j}}\left|L_{j k}\right|^{2}=\sum_{j, k} e^{g_{j}} f^{2}\left(g_{j}-g_{k}\right)\left|\dot{G}_{j k}\right|^{2} .
$$

As a simple example, we discuss the SLD and Fisher information for a qubit. Letting the Pauli matrices be denoted by $\sigma_{j}$, we can, without loss of generality, assume that the qubit state is diagonal in the eigenbasis of $\sigma_{3}$ and write the state as $\rho=\frac{1}{2}\left(\sigma_{0}+\sigma_{3} \tanh \gamma\right)$, where $\tanh \gamma$ is the expectation value of $\sigma_{3}$. This gives us

$$
\begin{aligned}
& G=\gamma \sigma_{3}-\ln (2 \cosh \gamma) \sigma_{0} \\
& \dot{G}=\dot{\gamma}\left(\sigma_{3}-\sigma_{0} \tanh \gamma\right)+\tau_{1} \sigma_{1}+\tau_{2} \sigma_{2} .
\end{aligned}
$$

Here $\dot{\gamma}$ accounts for the change in the eigenvalues of $\rho$ as $\theta$ changes, and the real parameters $\tau_{1}$ and $\tau_{2}$ account for the change in eigenbasis of $\rho$ as $\theta$ changes. Putting Eqs. (2.16) and (2.17) into Eq. (2.6) or (2.12), we have

$$
L=\dot{\gamma}\left(\sigma_{3}-\sigma_{0} \tanh \gamma\right)+\frac{\tanh \gamma}{\gamma}\left(\tau_{1} \sigma_{1}+\tau_{2} \sigma_{2}\right)
$$


This expression can be verified by expanding the $2 \times 2$ density operator explicitly. The result for the Fisher information is

$$
\mathcal{I}=\frac{\dot{\gamma}^{2}}{\cosh ^{2} \gamma}+\frac{\tanh ^{2} \gamma}{\gamma^{2}}\left(\tau_{1}^{2}+\tau_{2}^{2}\right)
$$

When the eigenvalues of the density operator $\rho$ are independent of $\theta$, i.e., the change of $\rho$ can be described by a unitary process, we have $\dot{G}=i[G, H]=i \mathcal{C}(H)$, where $H$ is some Hermitian operator. Putting this expression into Eq. (2.6), we have the following formula for the SLD:

$$
L=f(\mathcal{C})(\dot{G})=i f(\mathcal{C}) \mathcal{C}(H)=2 i \tanh (\mathcal{C} / 2)(H)
$$

which was first found by Knysh and Durkin (see Eq. (A3) of Ref. [25]).

\section{GAUSSIAN STATES IN EXPONENTIAL FORM}

In this section, we apply the expansion (2.6) to Gaussian states, which naturally adopt the exponential form,

$$
\rho=e^{G}=\exp \left(-\frac{1}{2} \mathbf{r}^{T} \Omega \mathbf{r}+\mathbf{r}^{T} \boldsymbol{\eta}-\ln Z\right)
$$

where $\mathbf{r}=\left(\begin{array}{llllll}x_{1} & \cdots & x_{n} & p_{1} & \cdots & p_{n}\end{array}\right)^{T}$ is the $2 n$-dimensional vector of position and momentum operators, $\boldsymbol{\eta}$ is a real $2 n$-dimensional vector, and $\Omega>0$ is a $2 n \times 2 n$ real, symmetric matrix. The state (3.1) can be regarded as a thermal state, with $\beta=1$, of the quadratic Hamiltonian

$$
H=\frac{1}{2} \mathbf{r}^{T} \Omega \mathbf{r}-\mathbf{r}^{T} \boldsymbol{\eta}
$$

notice that $Z=\operatorname{tr}\left(e^{-H}\right)$.

The canonical commutation relations can be written as $\left[r_{j}, r_{k}\right]=i J_{j k}$, where $J$ is the skew-symmetric matrix

$$
J=\left(\begin{array}{cc}
0 & \mathbb{1} \\
-\mathbb{1} & 0
\end{array}\right)=-J^{T}=-J^{-1},
$$

with $\mathbb{1}$ being the $n \times n$ identity matrix. For any Gaussian state, both $G$ and $\dot{G}$ are degree- 2 polynomials of the position and momentum operators, and thus so are all the recursive commutators in Eq. (2.6). Consequently, $L$ is also a degree-2 polynomial of the position and momentum operators,

$$
L=\mathbf{r}^{T} \Phi \mathbf{r}+\mathbf{r}^{T} \boldsymbol{\zeta}-\nu
$$

where $\zeta$ is a real $2 n$-dimensional vector, and $\Phi$ is a $2 n \times 2 n$ real, symmetric matrix, and $\nu$ can be determined by the trace-preserving condition,

$$
\nu=\operatorname{tr}\left(\rho \mathbf{r}^{T} \Phi \mathbf{r}\right)
$$

In order to use the expansion (2.6) efficiently, we write the quadratic Hamiltonian in the basis of creation and annihilation operators,

$$
H=\frac{1}{2} \overline{\boldsymbol{a}} \Omega^{\prime} \boldsymbol{a}-\overline{\boldsymbol{a}} \boldsymbol{\eta}^{\prime}
$$

where $\boldsymbol{a}$ and $\overline{\boldsymbol{a}}$ are vectors of the creation and annihilation operators,

$$
\begin{aligned}
& \overline{\boldsymbol{a}}=\left(a_{1}^{\dagger} \cdots a_{n}^{\dagger} a_{1} \cdots a_{n}\right), \\
& \boldsymbol{a}=\left(a_{1} \cdots a_{n} a_{1}^{\dagger} \cdots a_{n}^{\dagger}\right)^{T}
\end{aligned}
$$

with $a_{j}=\left(x_{j}+i p_{j}\right) / \sqrt{2}$; the matrix $\Omega^{\prime}$ and the vector $\boldsymbol{\eta}^{\prime}$ satisfy

$$
\Omega^{\prime}=V^{\dagger} \Omega V, \quad \boldsymbol{\eta}^{\prime}=V^{\dagger} \boldsymbol{\eta}
$$


where $V$ is a unitary matrix linking the two bases, i.e., $V \boldsymbol{a}=\mathbf{r}$, or equivalently, $V^{\dagger} \mathbf{r}=\boldsymbol{a}$,

$$
V^{\dagger}=\frac{1}{\sqrt{2}}\left(\begin{array}{cc}
\mathbb{1} & i \mathbb{1} \\
\mathbb{1} & -i \mathbb{1}
\end{array}\right)
$$

Similarly, we can write the SLD as

$$
L=\overline{\boldsymbol{a}} \Phi^{\prime} \boldsymbol{a}+\overline{\boldsymbol{a}} \boldsymbol{\zeta}^{\prime}-\nu
$$

where $\Phi^{\prime}=V^{\dagger} \Phi V$, and $\boldsymbol{\zeta}^{\prime}=V^{\dagger} \boldsymbol{\zeta}$.

Without affecting the Fisher information, which is invariant under unitary transformations, we can displace the state (3.1) so that $\boldsymbol{\eta}=0$. Moreover, we now assume that the matrix $\Omega$ is in the diagonal form,

$$
\Omega=\left(\begin{array}{cc}
\operatorname{diag}\left(\epsilon_{1}, \ldots, \epsilon_{n}\right) & 0 \\
0 & \operatorname{diag}\left(\epsilon_{1}, \ldots, \epsilon_{n}\right)
\end{array}\right)=\Omega^{\prime},
$$

which gives

$$
G=-H-\ln Z=-\sum_{j=1}^{n} \epsilon_{j}\left(a_{j}^{\dagger} a_{j}+\frac{1}{2}\right)-\ln Z .
$$

This case is important, because any Gaussian state is equivalent to it up to a Gaussian unitary, i.e., a symplectic transformation of the creation and annihilation operators. The commutation relations between $G$ and the creation and annihilation operators are straightforward:

$$
\left[G, a_{j}\right]=\epsilon_{j} a_{j}, \quad\left[G, a_{j}^{\dagger}\right]=-\epsilon_{j} a_{j}^{\dagger} .
$$

Consequently, we have

$$
f(\mathcal{C})\left(a_{j}\right)=f\left(\epsilon_{j}\right) a_{j}, \quad f(\mathcal{C})\left(a_{j}^{\dagger}\right)=f\left(\epsilon_{j}\right) a_{j}^{\dagger},
$$

and for quadratic operators, we have

$$
\begin{aligned}
& f(\mathcal{C})\left(a_{j}^{\dagger} a_{k}\right)=f\left(\epsilon_{k}-\epsilon_{j}\right) a_{j}^{\dagger} a_{k}, \\
& f(\mathcal{C})\left(a_{j} a_{k}\right)=f\left(\epsilon_{j}+\epsilon_{k}\right) a_{j} a_{k} .
\end{aligned}
$$

Most generally, the derivative of $G$ takes the form

$$
\dot{G}=-\frac{1}{2} \overline{\boldsymbol{a}} \dot{\Omega}^{\prime} \boldsymbol{a}+\overline{\boldsymbol{a}} \dot{\boldsymbol{\eta}}^{\prime}-\frac{\dot{Z}}{Z},
$$

Putting Eq. (3.18) into Eq. (2.6) and using the relations (3.15)-(3.17), we have

$$
\nu=\dot{Z} / Z, \quad \boldsymbol{\zeta}_{j}^{\prime}=f\left(\epsilon_{j}\right) \dot{\boldsymbol{\eta}}_{j}^{\prime}
$$

and

$$
\Phi_{j k}^{\prime}= \begin{cases}-\frac{1}{2} f\left(\epsilon_{j}-\epsilon_{k}\right) \dot{\Omega}_{j k}^{\prime}, & \text { for } j, k \leq n \text { or } j, k>n \\ -\frac{1}{2} f\left(\epsilon_{j}+\epsilon_{k}\right) \dot{\Omega}_{j k}^{\prime}, & \text { for all other cases }\end{cases}
$$

where $\epsilon_{j+n}=\epsilon_{j}$ for $j \leq n$. Equations (3.19) and (3.20) are explicit, and the only work required is to find the basis of the creation and annihilation operators, by a symplectic transformation, so that the Gaussian state is of the diagonal form (3.13).

Knowing the SLD allows one to calculate the Fisher information [see Eq. (4.29)],

$$
\mathcal{I}=\frac{1}{2} \operatorname{tr}(\Gamma \Phi \Gamma \Phi)-\frac{1}{2}\left(J \Phi J^{T} \Phi\right)+\frac{1}{2} \zeta^{T} \Gamma \zeta
$$

where $\Gamma$ is the covariance matrix of the Gaussian state defined in Eq. (4.3). Going to the basis of creation and annihilation operators, we have

$$
\mathcal{I}=\frac{1}{2} \operatorname{tr}\left(\Gamma^{\prime} \Phi^{\prime} \Gamma^{\prime} \Phi^{\prime}\right)-\frac{1}{2}\left(J^{\prime} \Phi^{\prime} J^{\prime} \Phi^{\prime}\right)+\frac{1}{2} \zeta^{\prime \dagger} \Gamma^{\prime} \zeta^{\prime}
$$


where

$$
J^{\prime}=J^{\dagger}=\left(\begin{array}{cc}
\mathbb{1} & 0 \\
0 & -\mathbb{1}
\end{array}\right),
$$

and for $\Omega^{\prime}$ taking the form (3.12), we have $\Gamma^{\prime}=V^{\dagger} \Gamma V=\operatorname{coth}\left(\Omega^{\prime} / 2\right)$. Thus, the Fisher information can be calculated explicitly,

$$
\mathcal{I}=\sum_{j, k=1}^{n}\left(\left|\Phi_{j k}^{\prime}\right|^{2}+\left|\Phi_{j, k+n}^{\prime}\right|^{2}\right) \operatorname{coth} \frac{\epsilon_{j}}{2} \operatorname{coth} \frac{\epsilon_{k}}{2}+\left|\Phi_{j, k+n}^{\prime}\right|^{2}-\left|\Phi_{j k}^{\prime}\right|^{2}+\sum_{j=1}^{n}\left|\zeta_{j}^{\prime}\right|^{2} \operatorname{coth} \frac{\epsilon_{j}}{2}
$$

\section{GAUSSIAN STATES BY MOMENTS}

A number of authors have already discussed SLDs and quantum Fisher information for Gaussian states. Monras and Paris [26] investigated the problem of loss estimation with displaced squeezed thermal states. Pinel et al. [27, 28] discussed parameter estimation with pure Gaussian states of arbitrarily many modes and general single-mode Gaussian states. Recently, Monras [29] found an equation - in terms of the moments - for the SLD of the most general Gaussian state. The Fisher information can be calculated once the SLD is known. Here we confirm Monras' results by using a different, somewhat simpler approach. Furthermore, we solve the resultant equation of the SLD with a symplectic transformation. Special cases are also discussed, which include pure, degenerate, and very noisy Gaussian states.

Most generally, the symmetrically ordered characteristic function of a Gaussian quantum state takes the form

$$
\chi_{S}(\boldsymbol{\xi}) \equiv \operatorname{tr}\left(\rho e^{i \mathbf{r}^{T} \boldsymbol{\xi}}\right)=\exp \left(-\frac{1}{4} \boldsymbol{\xi}^{T} \Gamma \boldsymbol{\xi}+i \boldsymbol{\delta}^{T} \boldsymbol{\xi}\right)
$$

where $\boldsymbol{\delta}$ is a real $2 N$-dimensional vector, and $\Gamma>0$ is a $2 N \times 2 N$ real, symmetric matrix. The vector $\boldsymbol{\delta}$ and the matrix $\Gamma$ represent the means and the covariance matrix of the Gaussian state,

$$
\begin{aligned}
& \delta_{j}=\operatorname{tr}\left(\rho r_{j}\right), \\
& \Gamma_{j k}=\operatorname{tr}\left(\rho\left\{\Delta r_{j}, \Delta r_{k}\right\}\right),
\end{aligned}
$$

where $\Delta r_{j}=r_{j}-\delta_{j}$. Without loss of generality, the mean $\boldsymbol{\delta}$ can be removed by a displacement,

$$
\rho \rightarrow e^{-i \mathbf{r}^{T} J \boldsymbol{\delta}} \rho e^{i \mathbf{r}^{T} J \boldsymbol{\delta}},
$$

and we assume $\boldsymbol{\delta}=0$ from now on.

\section{A. Calculating the SLD}

Taking a derivative with respect to $\theta$ on both sides of Eqs. (4.2) and (4.3) and using the definition (1.3), we have

$$
\begin{aligned}
& \dot{\delta}_{j}=\frac{1}{2} \operatorname{tr}\left(\{\rho, L\} r_{j}\right)=\frac{1}{2} \operatorname{tr}\left(\rho\left\{L, r_{j}\right\}\right), \\
& \dot{\Gamma}_{j k}=\frac{1}{2} \operatorname{tr}\left(\{\rho, L\}\left\{r_{j}, r_{k}\right\}\right)=\frac{1}{2} \operatorname{tr}\left(\rho\left\{L,\left\{r_{j}, r_{k}\right\}\right\}\right) .
\end{aligned}
$$

To calculate the traces in Eqs. (4.5) and (4.6), we introduce the following function, which we call the partially symmetrically ordered characteristic function,

$$
\begin{aligned}
\chi_{P}\left(\boldsymbol{\xi}_{1}, \boldsymbol{\xi}_{2}\right) & \equiv \frac{1}{2} \operatorname{tr}\left(\rho\left\{e^{i \mathbf{r}^{T} \boldsymbol{\xi}_{1}}, e^{i \mathbf{r}^{T} \boldsymbol{\xi}_{2}}\right\}\right) \\
& =\chi_{S}\left(\boldsymbol{\xi}_{1}+\boldsymbol{\xi}_{2}\right) \cos \left(\frac{1}{2} \boldsymbol{\xi}_{1}^{T} J \boldsymbol{\xi}_{2}\right) .
\end{aligned}
$$

Denoting the partial derivative with respect to the $j$ th element of $\boldsymbol{\xi}_{1,2}$ by $\partial_{j}^{(1,2)}$ we have

$$
\begin{aligned}
& \dot{\delta}_{j}=-\left.i \mathcal{L}^{(1)} \partial_{j}^{(2)} \chi_{P}\right|_{\boldsymbol{\xi}_{1}=\boldsymbol{\xi}_{2}=0}, \\
& \dot{\Gamma}_{j k}=-\left.2 \mathcal{L}^{(1)} \partial_{j k}^{(2)} \chi_{P}\right|_{\boldsymbol{\xi}_{1}=\boldsymbol{\xi}_{2}=0},
\end{aligned}
$$


where $\partial_{j k}=\partial_{j} \partial_{k}$ and

$$
\mathcal{L}=-\sum_{m, n} \Phi_{m n} \partial_{m n}-i \sum_{l} \zeta_{l} \partial_{l}-\nu
$$

Putting Eq. (4.10) into Eqs. (4.8) and (4.9), we have

$$
\begin{aligned}
\dot{\delta}_{j} & =-\left.\left(\sum_{l} \zeta_{l} \partial_{l}^{(1)}\right) \partial_{j}^{(2)} \chi_{P}\right|_{\boldsymbol{\xi}_{1,2}=0}=\frac{1}{2} \sum_{l} \Gamma_{j l} \zeta_{l}, \\
\dot{\Gamma}_{j k} & =\left.2\left(\sum_{m, n} \Phi_{m n} \partial_{m n}^{(1)}+\nu\right) \partial_{j k}^{(2)} \chi_{P}\right|_{\boldsymbol{\xi}_{1,2}=0} \\
& =\left(\frac{1}{2} \operatorname{tr}(\Gamma \Phi)-\nu\right) \Gamma_{j k}+(\Gamma \Phi \Gamma+J \Phi J)_{j k},
\end{aligned}
$$

where all the odd-order derivatives are neglected, because they vanish at $\boldsymbol{\xi}_{1}=\boldsymbol{\xi}_{2}=0$ for $\boldsymbol{\delta}=0$. By using the trace-preserving condition,

$$
0=\operatorname{tr}(L \rho)=\left.\mathcal{L} \chi_{S}\right|_{\xi=0}=\frac{1}{2} \operatorname{tr}(\Gamma \Phi)-\nu
$$

we have the following matrix forms

$$
\begin{aligned}
\dot{\boldsymbol{\delta}} & =\frac{1}{2} \Gamma \boldsymbol{\zeta}, \\
\dot{\Gamma} & =\Gamma \Phi \Gamma-J \Phi J^{T},
\end{aligned}
$$

Equation (4.15) is an implicit matrix equation, which is generally hard to solve. A way to circumvent such difficulty is by using a symplectic transformation. Any covariance matrix $\Gamma$ can be brought into the following standard (canonical) form by a symplectic transformation $S$ satisfying $S J S^{T}=J$,

$$
S \Gamma S^{T}=\Gamma_{\mathrm{s}}=\left(\begin{array}{cc}
\Lambda & 0 \\
0 & \Lambda
\end{array}\right)
$$

where $\Lambda=\operatorname{diag}\left(\lambda_{1}, \lambda_{2}, \ldots, \lambda_{n}\right) \geq \mathbb{1}$ is a diagonal matrix (equality holds, i.e., $\lambda_{j}=1$ for $j=1, \ldots, n$, only for pure states). In the basis that $\Gamma$ is standard, Eq. (4.15) reads

$$
\dot{\Gamma}_{\mathrm{s}}=\Gamma_{\mathrm{s}} \Phi_{\mathrm{s}} \Gamma_{\mathrm{s}}-J \Phi_{\mathrm{s}} J^{T}
$$

where $\dot{\Gamma}_{\mathrm{s}}=S \dot{\Gamma} S^{T}$, and $J \Phi_{\mathrm{s}} J^{T}=S J \Phi J^{T} S^{T}$. Noticing that $\Gamma_{\mathrm{s}}$ and $J$ commute, we have

$$
\Gamma_{\mathrm{s}} \dot{\Gamma}_{\mathrm{s}} \Gamma_{\mathrm{s}}+J \dot{\Gamma}_{\mathrm{s}} J^{T}=\Gamma_{\mathrm{s}}^{2} \Phi_{\mathrm{s}} \Gamma_{\mathrm{s}}^{2}-\Phi_{\mathrm{s}}
$$

which can be solved explicitly since $\Gamma_{\mathrm{s}}$ is diagonal,

$$
\left(\Phi_{\mathrm{s}}\right)_{j k}=\frac{\left(\Gamma_{\mathrm{s}} \dot{\Gamma}_{\mathrm{s}} \Gamma_{\mathrm{s}}+J \dot{\Gamma}_{\mathrm{s}} J^{T}\right)_{j k}}{\lambda_{j}^{2} \lambda_{k}^{2}-1}
$$

where $\lambda_{j+n}=\lambda_{j}$ for $j \leq n$. Once $\Phi_{\mathrm{s}}$ is determined in terms of $\Gamma_{\mathrm{s}}$ and $\dot{\Gamma}_{\mathrm{s}}$, an inverse symplectic transformation can transform it back to $\Phi$. To end this subsection, we discuss some special cases where Eq. (4.19) can be simplified to forms which are manifestly symplectic covariant; this allows us to solve the SLD and the Fisher information without going to the standard basis.

For a very noisy Gaussian state where all $\lambda_{j} \gg 1$, we have the following relations

$$
\Phi_{\mathrm{s}} \approx \Gamma_{\mathrm{s}}^{-1} \dot{\Gamma}_{\mathrm{s}} \Gamma_{\mathrm{s}}^{-1},
$$

which is symplectic covariant and can be generalized to

$$
\Phi \approx \Gamma^{-1} \dot{\Gamma} \Gamma^{-1} .
$$


For the degenerate case where $\lambda_{j}=\lambda$ for all $j$, we have

$$
\Phi_{\mathrm{s}}=\frac{1}{\lambda^{4}-1}\left(\lambda^{2} \dot{\Gamma}_{\mathrm{s}}+J \dot{\Gamma}_{\mathrm{s}} J^{T}\right)
$$

which can be brought into the following symplectic covariant form,

$$
\Phi=\frac{1}{\lambda^{4}-1}\left(\lambda^{4} \Gamma^{-1} \dot{\Gamma} \Gamma^{-1}+J \dot{\Gamma} J^{T}\right)
$$

If the symplectic eigenvalues of $\Gamma$ do not change, i.e., $\dot{\Gamma}$ is driven by some Gaussian unitary, we have

$$
\dot{\Gamma}=\Gamma H J^{T}+J H \Gamma,
$$

where $H=H^{T}$; this equation can be derived by considering the evolution of the covariance matrix (4.3) under the quadratic Hamiltonian $\mathbf{r}^{T} H \mathbf{r} / 2$. With the condition $\lambda^{2} \Gamma^{-1}=J \Gamma J^{T}$ for degenerate Gaussian states and Eq. (4.24), we have

$$
\lambda^{2} \Gamma^{-1} \dot{\Gamma} \Gamma^{-1}=J \Gamma H+H \Gamma J^{T}=-J \dot{\Gamma} J^{T},
$$

and thus Eq. (4.23) can be simplified to

$$
\Phi=\frac{\lambda^{2}}{\lambda^{2}+1} \Gamma^{-1} \dot{\Gamma} \Gamma^{-1}=-\frac{1}{\lambda^{2}+1} J \dot{\Gamma} J^{T} .
$$

For pure Gaussian states $(\lambda=1)$, we assume that the condition Eq. (4.25) is always satisfied; otherwise, $\Phi$ would diverge according to Eq. (4.23). By setting $\lambda=1$ in Eq. (4.26), we have the following result for pure states:

$$
\Phi=\frac{1}{2} \Gamma^{-1} \dot{\Gamma} \Gamma^{-1}=-\frac{1}{2} J \dot{\Gamma} J^{T} .
$$

Note that Eq. (4.27) is valid even if the pure Gaussian state actually goes through a nonunitary process which gives the same $\dot{\rho}$ as a unitary process for that pure state.

\section{B. Quantum Fisher information}

The Fisher information can be calculated by applying $\mathcal{L}$ on $\chi_{P}$ twice,

$$
\mathcal{I}=\operatorname{tr}\left(\rho L^{2}\right)=\left.\mathcal{L}^{(1)} \mathcal{L}^{(2)} \chi_{P}\right|_{\boldsymbol{\xi}_{1,2}=0} .
$$

Putting Eq. (4.10) into Eq. (4.28) and neglecting all derivatives of odd orders, we have

$$
\begin{aligned}
\mathcal{I} & =\left.\left(\sum_{j, k, l, m} \Phi_{j k} \Phi_{l m} \partial_{j k}^{(1)} \partial_{l m}^{(2)}+\sum_{j, k} 2 \nu \Phi_{j k} \partial_{j k}^{(1)}-\sum_{j, k} \zeta_{j} \zeta_{k} \partial_{j}^{(1)} \partial_{k}^{(2)}+\nu^{2}\right) \chi_{P}\right|_{\boldsymbol{\xi}_{1,2}=0} \\
& =\frac{1}{2} \operatorname{tr}(J \Phi J \Phi)+\frac{1}{2} \operatorname{tr}(\Gamma \Phi \Gamma \Phi)+\frac{1}{4}(\operatorname{tr}(\Gamma \Phi))^{2}-\nu \operatorname{tr}(\Gamma \Phi)+\frac{1}{2} \boldsymbol{\zeta}^{T} \Gamma \boldsymbol{\zeta}+\nu^{2} \\
& =\frac{1}{2} \operatorname{tr}\left(\left(\Gamma \Phi \Gamma-J \Phi J^{T}\right) \Phi\right)+\frac{1}{2} \boldsymbol{\zeta}^{T} \Gamma \boldsymbol{\zeta} \\
& =\frac{1}{2} \operatorname{tr}(\dot{\Gamma} \Phi)+2 \dot{\boldsymbol{\delta}}^{T} \Gamma^{-1} \dot{\boldsymbol{\delta}}
\end{aligned}
$$

where the conditions (4.13), (4.14), and (4.15) are used to simplify the expressions; also note that the quantity $\operatorname{tr}(\dot{\Gamma} \Phi)$ is symplectically invariant, specifically,

$$
\operatorname{tr}(\dot{\Gamma} \Phi)=\operatorname{tr}\left(\dot{\Gamma}_{\mathrm{s}} \Phi_{\mathrm{s}}\right)
$$


For very noisy Gaussian states, we have $\dot{\Gamma} \Phi=\left(\dot{\Gamma} \Gamma^{-1}\right)^{2}$ by Eq. (4.21), and consequently, the quantum Fisher information reads

$$
\mathcal{I}_{\text {noisy }} \approx \frac{1}{2} \operatorname{tr}\left(\left(\dot{\Gamma} \Gamma^{-1}\right)^{2}\right)+2 \dot{\boldsymbol{\delta}}^{T} \Gamma^{-1} \dot{\boldsymbol{\delta}} .
$$

For a degenerate Gaussian state, the quantum Fisher information can be derived by using Eq. (4.23),

$$
\mathcal{I}_{\text {degen }}=\frac{\operatorname{tr}\left(\lambda^{4}\left(\dot{\Gamma} \Gamma^{-1}\right)^{2}-(\dot{\Gamma} J)^{2}\right)}{2\left(\lambda^{4}-1\right)}+2 \dot{\boldsymbol{\delta}}^{T} \Gamma^{-1} \dot{\boldsymbol{\delta}}
$$

If the degenerate Gaussian state is driven by a Gaussian unitary, we have

$$
\mathcal{I}_{\text {degen }}=\frac{\lambda^{2}}{2\left(\lambda^{2}+1\right)} \operatorname{tr}\left(\left(\dot{\Gamma} \Gamma^{-1}\right)^{2}\right)+2 \dot{\boldsymbol{\delta}}^{T} \Gamma^{-1} \dot{\boldsymbol{\delta}}
$$

or equivalently,

$$
\mathcal{I}_{\text {degen }}=\frac{1}{2\left(\lambda^{2}+1\right)} \operatorname{tr}\left((\dot{\Gamma} J)^{2}\right)+\frac{2}{\lambda^{2}} \dot{\boldsymbol{\delta}}^{T} J \Gamma J^{T} \dot{\boldsymbol{\delta}}
$$

where we use the identity $\Gamma^{-1}=J \Gamma J^{T} / \lambda^{2}$ for degenerate Gaussian states. In particular, Eqs. (4.33) and (4.34) work for all single-mode Gaussian states.

For pure Gaussian states, we have

$$
\mathcal{I}_{\text {pure }}=\frac{1}{4} \operatorname{tr}\left(\left(\dot{\Gamma} \Gamma^{-1}\right)^{2}\right)+2 \dot{\boldsymbol{\delta}}^{T} \Gamma^{-1} \dot{\boldsymbol{\delta}}
$$

which coincides with Eq. (8) in [27], or equivalently,

$$
\mathcal{I}_{\text {pure }}=\frac{1}{4} \operatorname{tr}\left((\dot{\Gamma} J)^{2}\right)+2 \dot{\boldsymbol{\delta}}^{T} J \Gamma J^{T} \dot{\boldsymbol{\delta}}
$$

\section{CONCLUSION}

For a quantum state in exponential form, we give expressions for the SLD, see Eqs. (2.6) and (2.12), and the quantum Fisher information, see Eq. (2.15). All these expressions are explicit and are useful for quantum-metrology problems with Gaussian or general thermal states (but are not restricted to these two kinds of states). We give the quantum Fisher information, see Eq. (3.24), for a Gaussian state in terms of its generator. Using a different approach, we derive an equation for the SLD of an arbitrary Gaussian state in terms of its moments, confirming a recent result by Monras [29]. We find that the resulting equation is symplectic-covariant and can be solved exactly in the basis where the covariance matrix is in the standard form. Furthermore, the Fisher information in terms of the moments of a general Gaussian state is calculated; special cases are discussed, which include pure, degenerate, and very noisy Gaussian states.

\section{ACKNOWLEDGMENTS}

Special thanks go to C. M. Caves for his very useful advice. The author also thanks J. Combes, M. D. Lang, and I. H. Deutsch for helpful and enlightening discussions. This work was supported by National Science Foundation Grant Nos. PHY-1314763 and PHY-1212445 and Office of Naval Research Grant No. N00014-11-1-0082.

[1] T. Udem, R. Holzwarth, and T. W. Hänsch, "Optical frequency metrology," Nature 416, 233 (2002).

[2] N. Hinkley, J. A. Sherman, N. B. Phillips, M. Schioppo, N. D. Lemke, K. Beloy, M. Pizzocaro, C. W. Oates, and A. D. Ludlow, "An atomic clock with $10^{-18}$ instability," Science 341, 1215 (2013).

[3] LIGO Scientific Collaboration, "Enhanced sensitivity of the LIGO gravitational wave detector by using squeezed states of light," Nature Photonics 7, 613 (2013). 
[4] U. L. Andersen, "Quantum optics: Squeezing more out of LIGO," Nature Photonics 7, 589 (2013).

[5] J. A. Dunningham and K. Burnett, "Sub-shot-noise-limited measurements with Bose-Einstein condensates," Physical Review A 70, 033601 (2004).

[6] M. F. Riedel, P. Böhi, Y. Li, T. W. Hänsch, A. Sinatra, and P. Treutlein, "Atom-chip-based generation of entanglement for quantum metrology," Nature 464, 1170 (2010).

[7] J. R. Maze, P. L. Stanwix, J. S. Hodges, S. Hong, J. M. Taylor, P. Cappellaro, L. Jiang, M. V. G. Dutt, E. Togan, A. S. Zibrov, A. Yacoby, R. L. Walsworth, and M. D. Lukin, "Nanoscale magnetic sensing with an individual electronic spin in diamond," Nature 455, 644 (2008).

[8] J. M. Taylor, P. Cappellaro, L. Childress, L. Jiang, D. Budker, P. R. Hemmer, A. Yacoby, R. Walsworth, and M. D. Lukin, "High-sensitivity diamond magnetometer with nanoscale resolution," Nature Physics 4, 810 (2008).

[9] S. Boixo, S. T. Flammia, C. M. Caves, and J. M. Geremia, "Generalized limits for single-parameter quantum estimation," Physical Review Letters 98, 090401 (2007).

[10] M. Tsang, H. M. Wiseman, and C. M. Caves, "Fundamental quantum limit to waveform estimation," Physical Review Letters 106, 090401 (2011).

[11] B. M. Escher, R. L. de Matos Filho, and L. Davidovich, "General framework for estimating the ultimate precision limit in noisy quantum-enhanced metrology," Nature Physics 7, 406 (2011).

[12] V. Giovannetti, S. Lloyd, and L. Maccone, "Quantum measurement bounds beyond the uncertainty relations," Physical Review Letters 108, 260405 (2012).

[13] http://en.wikipedia.org/wiki/Fisher_information.

[14] R. A. Fisher, "Theory of statistical estimation," Mathematical Proceedings of the Cambridge Philosophical Society 22, 700 (1925).

[15] H. Cramér, Mathematical methods of statistics (Princeton University Press, Princeton, 1999).

[16] S. L. Braunstein and C. M. Caves, "Statistical distance and the geometry of quantum states," Physical Review Letters 72, 3439 (1994).

[17] C. W. Helstrom, Quantum detection and estimation theory (Academic Press, 1976).

[18] A. S. Holevo, Probabilistic and statistical aspects of quantum theory (North-Holland Pub. Co., Amsterdam, 1982).

[19] S. L. Braunstein, C. M. Caves, and G. J. Milburn, "Generalized uncertainty relations: Theory, examples, and lorentz invariance," Annals of Physics 247, 135 (1996).

[20] M. Aspachs, J. Calsamiglia, R. Muñoz Tapia, and E. Bagan, "Phase estimation for thermal Gaussian states," Physical Review A 79, 033834 (2009).

[21] P. M. Anisimov, G. M. Raterman, A. Chiruvelli, W. N. Plick, S. D. Huver, H. Lee, and J. P. Dowling, "Quantum metrology with two-mode squeezed vacuum: Parity detection beats the Heisenberg limit," Physical Review Letters 104, 103602 (2010).

[22] M. D. Lang and C. M. Caves, "Optimal quantum-enhanced interferometry using a laser power source," Physical Review Letters 111, 173601 (2013).

[23] U. Marzolino and D. Braun, "Precision measurements of temperature and chemical potential of quantum gases," Phys. Rev. A 88, 063609 (2013).

[24] R. M. Wilcox, "Exponential operators and parameter differentiation in quantum physics," Journal of Mathematical Physics 8, 962 (1967).

[25] S. I. Knysh and G. A. Durkin, "Estimation of phase and diffusion: Combining quantum statistics and classical noise," arXiv:1307.0470 (2013).

[26] A. Monras and M. G. A. Paris, "Optimal quantum estimation of loss in bosonic channels," Physical Review Letters 98, 160401 (2007).

[27] O. Pinel, J. Fade, D. Braun, P. Jian, N. Treps, and C. Fabre, "Ultimate sensitivity of precision measurements with intense Gaussian quantum light: A multimodal approach," Physical Review A 85, 010101 (2012).

[28] O. Pinel, P. Jian, N. Treps, C. Fabre, and D. Braun, "Quantum parameter estimation using general single-mode Gaussian states," Phys. Rev. A 88, 040102 (2013).

[29] A. Monras, "Phase space formalism for quantum estimation of Gaussian states," arXiv:1303.3682 (2013). 